\title{
Etude Des Facteurs De Risque De L’obésité Chez Le Personnel Du CHUD/Borgou à Parakou (Bénin) en 2013
}

\section{CA Dovonou}

Département de Médecine et Spécialités Médicales, Faculté de Médecine, Université de Parakou. Service de Médecine Interne, Centre Hospitalier Universitaire Départemental du Borgou.

\section{F Gounongbe}

Département de Médecine et Spécialités Médicales,

Faculté de Médecine, Université de Parakou.

\section{AV Hinson}

Département de Santé publique, Faculté des Sciences de la Santé de Cotonou, Université d'Abomey-Calavi.

\section{CA Alassani}

Département de Médecine et Spécialités Médicales,

Faculté de Médecine, Université de Parakou.

\section{CA Attinsounon}

Département de Médecine et Spécialités Médicales, Faculté de Médecine, Université de Parakou. Service de Médecine Interne, Centre Hospitalier Universitaire Départemental du Borgou.

\section{FT Tognon}

Département de Médecine et Spécialités Médicales,

Faculté de Médecine, Université de Parakou.

\section{FV Guedou}

Service de Médecine Interne,

Centre Hospitalier Universitaire Départemental du Borgou

\section{A Azon-Kouanou \\ DM Zannou \\ F Houngbe}

Département de Médecine et Spécialités Médicales, Faculté des Sciences de la Santé de Cotonou, Université d'Abomey-Calavi.

\section{B Fayomi}

Département de Santé publique, Faculté des Sciences de la Santé de Cotonou, Université d'Abomey-Calavi. 


\section{Abstract}

The objective of this study was to determine the prevalence of obesity among employees CHUD in Parakou and risk factors in 2013.

Methods: This was a cross sectional study, descriptive analytical referred to place from 05 August to 05 September 2013. The study population consists of employees of CHUD in Parakou. Data were collected using a questionnaire and by anthropometric measures.

Results: The overall prevalence of overweight and obesity was $55.9 \%$. The sex ratio was 0.8 . The mean age of subjects was $37.2 \pm 9.0$ years. Factors associated with obesity were: female gender $\left(\mathrm{p}=10^{-11}\right)$, those aged 30-49 years $(p=0.04)$, subjects with a level of secondary education limited $(p=$ $0.01)$, subjects with a daily consumption and accidental alcohol $\left(\mathrm{p}=10^{-9}\right)$, snacking $(\mathrm{p}=0.00012)$.

Conclusion: Obesity prevention should involve the establishment and maintenance during the lifetime of healthy eating habits and regular physical activity.

Keywords: Prevalence, obesity, risk factors, CHUD in Parakou

\section{Résumé}

L'objectif de cette étude était de déterminer les prévalences du surpoids, de l'obésité et les facteurs de risque associés chez le personnel administratif et de santé du CHUD de Parakou en 2013.

Méthodes: Il s'agissait d'une étude transversale, descriptive à visée analytique déroulée du 05 août au 05 septembre 2013. La population d'étude était constituée des salariés du CHUD de Parakou. Les données avaient été collectées à l'aide d'un questionnaire et par des mesures anthropométriques.

Résultats : La prévalence globale du surpoids et de l'obésité était de 55,9\%. La sex-ratio était 0,8. L'âge moyen des sujets était de 37,2 $\pm 9,0$ ans. Les facteurs associés à l'obésité étaient: le sexe féminin $\left(\mathrm{p}=10^{-11}\right)$, les sujets âgés de 30-49 ans $(\mathrm{p}=0,04)$, les sujets ayant un niveau d'instruction limité au secondaire $(\mathrm{p}=0,01)$, les sujets ayant une consommation quotidienne et accidentelle d'alcool $\left(\mathrm{p}=10^{-9}\right)$, le grignotage $(\mathrm{p}=0,00012)$.

Conclusion : La prévention de l'obésité doit faire appel à la mise en place et au maintien durant la vie des habitudes alimentaires saines et d'une activité physique régulière.

Mots clés : Prévalence, obésité, facteurs de risque, CHUD de Parakou. 


\section{Introduction:}

L’Organisation Mondiale de la Santé considère l'obésité comme une pandémie qui touche aussi bien les pays industrialisés que les pays en développement [OMS, 2005]. La prévalence du surpoids et de l'obésité est en croissance partout dans le monde [OMS, 2005]. L'obésité est estimée à plus de 50\% au Koweït, en Libye, au Qatar, au Tonga et au Samoa [OCDE, 2005]. En France elle est de 14,5\%, au Canada 14,9 \%, en Grèce 21,9 \%, au Mexique 24,2\% et au Etats-Unis 30,6 \% [OCDE, 2005].

En milieu professionnel, l'obésité est aussi préoccupante. Elle est de $38,1 \%$ chez les travailleurs du port autonome d'Abidjan [Koffi NM et al, 2001]. À Cotonou au Bénin, des enquêtes sur les facteurs de risque des maladies non transmissibles, avaient rapporté une prévalence de $21,5 \%$ d'obésité chez les adultes [Acakpo A et al, 2000].

Les causes de l'excès pondéral sont le manque d'activités physiques, le comportement alimentaire, la sédentarité, le niveau socioculturel et la surconsommation d'alcool puis des facteurs génétiques, environnementaux et psycho-sociaux. A celles-ci Il faut ajouter des prédispositions biologiques individuelles liées à l'âge, le sexe et l'ethnie.

L'objectif de cette étude est de déterminer les prévalences du surpoids, de l'obésité et les facteurs associés chez le personnel administratif et soignant du CHUD de Parakou en 2013.

\section{Méthodes d'étude}

L'étude s'est déroulée dans tous les services administratifs et hospitaliers du CHUD de Parakou du 05 août au 05 septembre 2013. Il s'agissait d'une étude transversale, descriptive à visée analytique. Elle a porté sur l'ensemble des 435 agents administratif et de soins de l'hôpital. En dehors des femmes enceintes, tous les salariés (soignants, administratifs) présents dans le centre durant la période de l'enquête, ayant donné leur consentement éclairé, sont inclus. La collecte des données a été faite en deux phases :

- Entretien à l'aide d'un questionnaire a permis de collecter les variables sociodémographiques et économiques (le sexe, l'âge, l'ethnie, la situation matrimoniale, la profession, le revenu mensuel), les comportementales (habitudes alimentaires, la consommation du tabac, la consommation d'alcool), et les activités physiques.

- La mesure des paramètres anthropométriques (poids, taille et tour de taille) a été réalisée. L'indice de masse corporelle (IMC) a été calculé chez tout enquêté. Le seuil d'IMC $\geq 30 \mathrm{~kg} / \mathrm{m}^{2}$ est retenu pour définir l'obésité globale. Le seuil du tour de taille prise à hauteur de l'ombilic est $>102 \mathrm{~cm}$ pour l'homme et $>88 \mathrm{~cm}$ pour la femme est utilisé conformément aux critères de l’OMS pour détecter l'obésité abdominale. 
Le traitement des données a été fait par le logiciel EPI-INFO version 3.5.3. L'analyse des données a été faite selon les recommandations du STEPS. Les variables qualitatives sont exprimées en fréquences avec leur intervalle de confiance à 95\%, et les variables quantitatives en moyenne avec leur écart-type. Les proportions ont été comparées grâce au test de chi-deux (ou le test exact de Fisher selon le cas) et les moyennes grâce au test t de Student. Les variables considérées dans l'analyse étaient le sexe, l’âge, le niveau d'instruction, le type de consommation d'alcool, la consommation de boissons sucrées, le grignotage et le stress. L’analyse était univariée. Pour ces comparaisons un seuil de significativité de $5 \%$ est utilisé $(\mathrm{p}<0,05)$.

\section{Résultats}

\section{Prévalence du surpoids et de l'obésité chez le personnel du CHUD- Borgou en 2013}

Au total, 290 des 435 salariés du CHUD de Parakou ont été enquêtés soit $66,7 \%$ de taux de participation. Ils étaient 160 femmes et 130 hommes ce qui donnait une sex-ratio $(\mathrm{H} / \mathrm{F})$ égale à 0,8 . L'âge moyen des sujets était de 37,2 \pm 9,0 ans avec des extrêmes de 19 ans et 67 ans.

Parmi les enquêtés, 92 étaient en surpoids soit une prévalence de

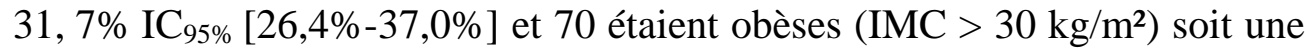
prévalence de $24,1 \% \mathrm{IC}_{95 \%}$ [19,2\%-29,0\%]. La proportion des femmes parmi les obèses $84,3 \%$ et celle des hommes était $15,7 \%$. L'obésité abdominale a été enregistrée chez 83 soit une prévalence de 28,6\% IC $95 \%$ [23,5\%-33,7\%].

\section{Comportements à risque d'obésité chez les enquêtés}

Quinze $(9,90 \%)$ des enquêtés consommaient quotidiennement de l'alcool et 29 (19,1\%) en faisaient une consommation nocive. Parmi les 290 sujets, 102 mangeaient régulièrement des aliments riches en graisse soit 35,5\% ; 123 (42,4\%) consommaient souvent les fibres. La consommation de boissons sucrées était régulière chez 95,9\%. Une proportion de $65,2 \%$ des enquêtés ne pratiquaient pas des activités physiques. Le reste des résultats était présenté dans les tableaux I à IV. Les tableaux I et II répartissaient les enquêtés en fonction de leurs caractéristiques socioprofessionnels, le tableau III présentait leurs comportements de vie, et le tableau IV donnait la prévalence de l’obésité en fonction des facteurs de risque en 2015. 
Tableau I : Répartition des enquêtés du CHUD-B selon les caractéristiques sociodémographiques en 2013

\begin{tabular}{|c|c|c|}
\hline & $\begin{array}{l}\text { Fréquence } \\
(\mathrm{N}=\mathbf{2 9 0})\end{array}$ & $\begin{array}{l}\text { Pourcentage } \\
(\%)\end{array}$ \\
\hline \multicolumn{3}{|l|}{ Age des enquêtés } \\
\hline$<20$ ans & 4 & 1,38 \\
\hline $20-29$ ans & 53 & 18,28 \\
\hline 30-39 ans & 123 & 42,41 \\
\hline 40-49 ans & 83 & 28,62 \\
\hline $50-59$ ans & 24 & 8,28 \\
\hline 60 ans et plus & 3 & 1,03 \\
\hline \multicolumn{3}{|l|}{ Niveau d'instruction } \\
\hline Analphabète & 8 & 2,75 \\
\hline Primaire & 57 & 19,66 \\
\hline Secondaire & 168 & 57,93 \\
\hline Supérieur & 57 & 19,66 \\
\hline \multicolumn{3}{|l|}{ Revenu mensuel (Fcfa) } \\
\hline Moins de 31.500 & 49 & 16,90 \\
\hline $31.500-100.000$ & 179 & 61,72 \\
\hline Plus de 100.000 & 62 & 21,38 \\
\hline \multicolumn{3}{|l|}{ Ethnie } \\
\hline Fon et apparentes & 134 & 46,2 \\
\hline Bariba & 49 & 16,9 \\
\hline Nago et apparentes & 46 & 15,9 \\
\hline Autres & 27 & 9,3 \\
\hline Dendi & 20 & 6,9 \\
\hline Lokpa/Yom & 9 & 3,1 \\
\hline Ditamari & 5 & 1,7 \\
\hline \multicolumn{3}{|l|}{ Situation matrimoniale } \\
\hline Marié /Concubinage & 231 & 79,7 \\
\hline Célibataire & 47 & 16,2 \\
\hline Divorcé ou veuf & 12 & 4,1 \\
\hline Tableau II : Répartition des sujets selon & $\begin{array}{l}\mathrm{t} \text { le statut profession } \\
\text { Effectifs (n) }\end{array}$ & $\begin{array}{l}\text { el au CHUD-B en } 201 \\
\text { Pourcentage (\%) }\end{array}$ \\
\hline \multicolumn{3}{|l|}{ Profession } \\
\hline Médecin & 14 & 04,82 \\
\hline Sage-femme & 04 & 01,38 \\
\hline Infirmier & 67 & 23,10 \\
\hline Technicien de laboratoire/Radiologie & 12 & 04,13 \\
\hline Assistant social & 02 & 00,70 \\
\hline Administrateur/Financier & 09 & 03,10 \\
\hline Aide-soignants & 92 & 31,72 \\
\hline Agent d'entretien de service & 39 & 13,44 \\
\hline Autres & 51 & 17,58 \\
\hline Total & 290 & 100,00 \\
\hline \multicolumn{3}{|l|}{ Statut professionnel } \\
\hline Agents permanents de l'Etat & 74 & 25,50 \\
\hline Agents contractuels de l'Etat & 127 & 43,80 \\
\hline Contractuels locaux du CHUD-B & 89 & 30,70 \\
\hline
\end{tabular}




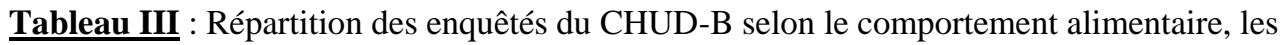
activités physiques et la durée de sommeil en 2015

\begin{tabular}{|c|c|c|}
\hline & Fréquence & Pourcentage (\%) \\
\hline \multicolumn{3}{|l|}{ Nombre de repas quotidiens } \\
\hline$<2$ & 03 & 01,03 \\
\hline 2 à 3 & 246 & 84,83 \\
\hline$>3$ & 41 & 14,14 \\
\hline \multicolumn{3}{|l|}{ Activités physiques $(n=74)$} \\
\hline Activités physiques de faible intensité & 32 & 43,24 \\
\hline Activités physiques modérées & 23 & 31,09 \\
\hline Activités physiques de forte intensité & 19 & 25,67 \\
\hline \multicolumn{3}{|c|}{ Durée quotidienne de sédentarité debout et/ou assis } \\
\hline$<6 \mathrm{H}$ & 228 & 78,60 \\
\hline$>6 \mathrm{H}$ & 62 & 21,40 \\
\hline \multicolumn{3}{|l|}{ Durée de sommeil au cours de la nuit } \\
\hline$<8 \mathrm{H}$ & 53 & 18,3 \\
\hline$>8 \mathrm{H}$ & 237 & 81,7 \\
\hline
\end{tabular}

Tableau IV : Répartition des facteurs de risque de l'obésité chez le personnel du CHUD-P en 2013

\begin{tabular}{lllll}
\hline & & \multicolumn{2}{l}{ Obésité } & \\
\cline { 4 - 5 } & Effectif total & Fréquence & \% & P \\
\hline Sexe & 160 & 59 & 36,87 & $10^{-11}$ \\
Femme & 130 & 11 & 08,46 & \\
Homme & & & & \\
Tranche d'âge & 04 & 00 & 00,00 & \\
$20-29$ ans & 53 & 6 & 11,32 & \\
$30-39$ ans & 123 & 29 & 23,57 & 0,04 \\
$40-49$ ans & 83 & 27 & 32,53 & \\
$50-59$ ans & 24 & 08 & 33,33 & \\
60 ans et plus & 03 & 00 & 00,00 & \\
Niveau d'instruction & & & & \\
Non scolarisé & 07 & 01 & 14,28 & \\
Primaire & 57 & 10 & 17,54 & 0,01 \\
Secondaire & 168 & 53 & 31,54 & \\
Supérieur & 57 & 06 & 10,52 & \\
Type de consommation & & & & \\
d'alcool & & & & \\
Quotidienne & 15 & 06 & 40,00 & \\
Nocive & 29 & 02 & 06,89 & $10^{-9}$ \\
Accidentelle & 108 & 62 & 57,40 & \\
Boissons sucrées & 278 & 68 & 24,46 & 0,44 \\
Grignotage & 117 & 31 & 26,5 & 0,00012 \\
Stress & 167 & 46 & 27,54 & 0,11 \\
\hline & & & & \\
\hline
\end{tabular}




\section{Discussion}

La présente étude nous a permis de contribuer à une meilleure connaissance de l’obésité au CHUD de Parakou en 2013. Elle a permis de déterminer la prévalence de l'obésité qui était de 24,1\% chez le personnel et d'en rechercher les facteurs de risques. Pour y arriver, nous avions réalisé une étude transversale, descriptive en vue de limiter les biais de sélection et d'assurer la représentativité de notre échantillon, ce qui est adapté pour l'atteinte des objectifs que nous nous sommes assignés.

Le sexe féminin prédominait avec une sex-ratio de 0,8. En 2011, Gounongbé ACF et al avaient déjà noté cette prédominance féminine (sexratio $=0,6$ ) chez le même personnel [Gounongbé FCA et al, 2013]. Il en était de même chez les travailleurs de santé à Cotonou en 2000 dont la sex-ratio était 0,95 [Acakpo A et al, 2000]. La prédominance féminine parmi le personnel de santé était constatée dans beaucoup de pays du monde [Gounongbé FCA et al, 2013].

En 2011, Gounongbé ACF et al [Gounongbé FCA et al, 2013] avaient déjà trouvé le personnel du CHUD de Parakou relativement jeune

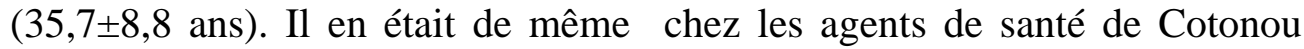
(31,3 \pm 7,2 ans) en 2000 [Acakpo A et al, 2000]. Cette relative jeunesse des agents du CHUD de Parakou est maintenue dans la présente étude (âge moyen $=37,2 \pm 9,0$ ans). Dans une série camerounaise en milieu de travail non sanitaire, Bita Fouda [Bita Fouda AA et al, 2012] rapporta un âge moyen de 34,5 \pm 6,8 ans en 2012. Par contre au Kosovo (2006), l'âge moyen des travailleurs était de $56 \pm 10$ ans [Soualem A et al, 2006], donc bien supérieur à celui de notre étude.

Près de la moitié des personnels du CHUD sont des contractuels de l'Etat. Environ trois sujets sur cinq ont un revenu mensuel entre 31.500100.000 FCFA; revenu supérieur ou égal au SMIG qui était 31.500. Le nombre important des aides-soignants dans l'échantillon expliquerait cet état de chose. Il s’en déduit que la plupart des salariés de cet Hôpital de Parakou ont des situations économiques très modestes. Les ressortissants du sud du Bénin (Fons, Nagos et apparentés) faisant 62\% de l'effectif, il va s'en dire que leur niveau de vie devrait être faible. La plupart des personnels étaient mariés (79,7\%). Ce taux est plus élevé que celui $(53,7 \%)$ trouvé par Janice BV et al [Janice BV et al, 2001] en Californie aux Etats-Unis en 2007. Ceci suggère que les personnels du CHUD seraient bien nourris. Le temps de travail étant huit heures d'affilées dans les hôpitaux du Bénin, amènerait à penser que les travailleurs de ces formations sanitaires grignotaient.

La prévalence globale de l’obésité était de 24,1\% chez les personnels du CHUD-Parakou en 2015. Cette prévalence est similaire à celle trouvée par Bita Fouda AA au Cameroun (23,4\%) [Bita Fouda AA et al, 2012], et à celle $(25,8 \%)$ rapportée chez les travailleurs postés dans une entreprise 
d'électricité en Tunisie [Chatti S et al, 2010]. Par contre elle est inférieure à la prévalence de l’obésité chez les hôteliers au Cameroun en 2006 [Etoundi Ngoa LS, 2006], et celle trouvée chez les travailleurs du port autonome d'Abidjan [Koffi NM et al, 2001] respectivement $37 \%$ et $38,1 \%$. L'environnement et les habitudes alimentaires très différentes de ces pays peuvent expliquer cette disparité entre les chiffres.

Les femmes sont plus affectées par la surcharge pondérale et l'obésité que les hommes [Pi-Sunyer FX et al, 2002]. Notre étude confirme cette affirmation. En effet, la prévalence de l'obésité obtenue chez les femmes (36,9\%) est plus élevée que chez les hommes $(8,5 \%)(\mathrm{P}<0,05)$. Le même constat a été fait par Bita Fouda AA et al.[ Bita Fouda AA et al, 2012] au Cameroun où l'obésité était présente chez 36,1\% des femmes contre 17,8\% des hommes $(\mathrm{P}<0,05)$; par Azankpan ER [Azankpan ER et al, 2010] en 2010 à Cotonou (84\% de femmes contre 16\% des hommes) ; par Bâ HO [Bâ HO et al, 2010] et al au Mali en 2008, par Dugas LR et al en Afrique du sud en 2009 [Dugas LR et al, 2009] où en milieu semi-urbain près de la moitié des femmes étaient en surpoids ou obèses alors qu'aucun homme ne l'était. Ceci éteigne bien la prédisposition biologique de la femme à l'obésité.

Des études ont conclu que l'obésité et la surcharge pondérale augmentent avec l’âge [Pi-Sunyer FX et al, 2002]. La tranche d'âge la plus représentée au CHUD de Parakou était celle de 40-49 ans suivie de celle de 50-59 ans soit respectivement 32,5\% et 33,3\%. Ces résultats se rapprochent de ceux du groupe de Janice BV et al. aux Etats-Unis (2001) qui avaient rapporté une prévalence de 34,19\% d’obésité chez les sujets âgés 50 à 64 ans [Janice BV et al, 2001]. Mais elle est un peu plus élevée que ceux d'Azankpan ER à Cotonou en 2010 qui était de 28\% dans la tranche d’âge 46-55 ans en population générale [Azankpan ER et al, 2010]. Les sujets ayant un niveau secondaire ont une prévalence de 31,5\% pour l'obésité. En 2000 à Cotonou, Acakpo et al. [Acakpo A et al, 2000] trouvèrent la prévalence la plus élevée de l'obésité chez les sujets ayant un niveau primaire (24,0\%). Mais à Cotonou, l'étude s'était déroulée en population générale alors que la nôtre s’est déroulée en milieu hospitalier où les enquêtés sont plus de la moitié de niveau secondaire ; de plus, un sujet sur cinq était de niveau supérieur.

\section{Conclusion:}

L’obésité est un problème de santé publique en milieu hospitalier dans la ville de Parakou, les femmes étant les plus à risque et les plus atteintes. Le sexe, l’âge, l'ethnie, le niveau d'étude, la situation matrimoniale, le revenu mensuel, la consommation excessive d'alcool, le régime alimentaire et le temps passé par journée en position fixe prolongée, sont les facteurs significativement associés à l’obésité. Au même moment, 
bien que le stress ne soit pas significativement associé à l'obésité, sa prévalence était élevée chez les sujets obèses. L’identification des facteurs de risque de l’obésité au Centre Hospitalier Universitaire Départemental du Borgou nécessite des actions pour un changement de comportement afin d'inverser la tendance actuelle. De plus, des études s’avèrent nécessaires en vue de rechercher chez ces travailleurs des complications associées à l’obésité.

\section{References:}

Organisation Mondiale de la Santé [Suisse]. Obésité: prévention et prise en charge de l’épidémie mondiale. Rapport technique. Genève : OMS, 2005; 894:15-65.

Organisation de coopération et de développement économique [Suisse]. Panorama de la Santé. Indicateurs de l’OCDE. Genève : OMS 2005:84-5.

Koffi NM, Sally SJ, Kouame P, Silue K, Diarra Nama AJ. Faciès de l'hypertension artérielle en milieu professionnel à Abidjan. Médecine d'Afrique Noire, 2001; 48:257-60.

Acakpo A, Fayomi B, Djrolo F, Kolanowski J, Agueh V, Makoutode M, et al. Prévalence et étude des facteurs déterminants de l'obésité à Cotonou. LOUVAIN MED 2000; 119:276-81.

Gounongbé FCA, Ayelo AP, Aguemon B, Chouti FL, Zannou MD, Fayomi B. Facteurs de risque des accidents d'exposition au sang chez les professionnels de la santé de la zone sanitaire Parakou-N'dali (Nord Bénin). Revue CAMES SANTÉ 2013; 1(1): 11-15.

Bita Fouda AA, Lemogoum D, Owona Manga J, Dissongo JII, Tobbit R, Ngounou Moyo DF et al. Epidemiology of obesity in the work milieu, Douala, Cameroon. Rev Med Brux 2012; 33:131-7.

Soualem A, Ahami AOT, Aboussaleh Y. Obesity and associated factors in Albanese adults of former Yougoslavia Antropo, 2006; 12:35-41.

Janice Bowie V, Hee-Soon J, Juhee CMA, Elisa Rodriguez M MS. Factors Associated With Overweight and Obesity Among Mexican Americans and Central Americans: Results From the 2001 California Health Interview Surveywww.cdc.gov/pcd/issues/2007/jan/06_0036.htm • Centers for Disease Control and Prevention.

Chatti S, Debbabi F, Ben AA, Harbaoui R, Ghannem H, Mrizak N. Facteurs de risque cardiovasculaire chez les travailleurs postés d'une centrale de production d'électricité au centre tunisien. Ann Cardiol Angeiol (Paris), 2010 ; 59 : 190-5.

Etoundi Ngoa LS, Melaman SF, Belinga A. Obésité en milieu hôtelier : Influence du poste de travail sur les métabolismes lipidique et glucidique et sur la pression artérielle de 80 employées de sexe féminin des hôtels de Yaoundé au Cameroun. Médecine d’Afrique Noire, 2006 ; 53 : 137-41. 
Pi-Sunyer FX. The epidemy of obesity: path physiology and consequences of obesity. Obesity Research 2002; 10:97-104.

Azankpan ER. Prévalence des facteurs de risque des accidents vasculaires cérébraux en population générale à Cotonou (Benin). Thèse de doctorat en médecine. Faculté de médecine, de pharmacie et d’odontologie (Sénégal) $2010 ; N^{\circ} 58$ 70-73P.

Bâ HO, Traoré A, Diarra MB, Maiga A, Diarra A, Diall IB et al. Facteurs de risque cardiovasculaire majeurs selon le sexe en milieu Hospitalier. Mali med 2010; 25(1):57-60.

Dugas LR, Carstens MA, Ebersole K, Schoeller DA, Durazo-Arvizu RA, Lambert EV, et al. Energy expenditure in young adult urban informal settlement dwellers in south Africa. Eur J Clin Nut 2009; 63(6):805-7 